\title{
Relations entre composition chimique, activité antioxydante et toxicité des huiles essentielles de deux espèces de Cymbopogon acclimatées au Bénin
}

\author{
Didier KPADONOU ${ }^{1}$, Florence ALLANTO ${ }^{1}$, Bénédicta KPADONOU-KPOVIESSI ${ }^{1,2}$, \\ Pierre AGBANI ${ }^{3}$, Fernand GBAGUIDI ${ }^{1,2}$, Lamine BABA-MOUSSA ${ }^{4}$, Joachim \\ GBENOU $^{2}$, Mansourou MOUDACHIROU ${ }^{2}$ et Salomé KPOVIESSI ${ }^{1,2,5 *}$ \\ ${ }^{1}$ Laboratoire de Chimie Organique Physique et de Synthèse (LaCOPS) de la Faculté des Sciences et \\ Techniques (FAST), de l'Université d'Abomey-calavi (UAC), 01 BP: 4166 Cotonou, Bénin. \\ ${ }^{2}$ Laboratoire de Pharmacognosie et des Huiles Essentielles (LAPHE) de la Faculté des Sciences de la Santé \\ (FSS), de l'Université d'Abomey-calavi (UAC). 01BP: 188 Cotonou, Bénin. \\ ${ }^{3}$ Laboratoire d'Ecologie Appliquée (LEA) de la Faculté des Sciences Agronomiques (FSA), de l'Université \\ d'Abomey-calavi (UAC). 03 BP: 1974 Cotonou, Bénin. \\ ${ }^{4}$ Laboratoire de Biologie et de Typage Moléculaire en Microbiologie (LBTMM) de FAST/UAC-Bénin. \\ ${ }^{5}$ Laboratoire du Groupe de Recherche Pharmacognosie, Louvain Drug Research Institute (LDRI) de \\ l'Université Catholique de Louvain (UCL). B1 7203 Av. E. Mounier 72, B-1200 Bruxelles, Belgique. \\ *Auteur correspondant ; E-mail: kpovsalome@yahoo.fr; Tel: +229 97883927.
}

\section{RESUME}

Les plantes aromatiques jouent un rôle considérable dans plusieurs secteurs notamment dans la médicine traditionnelle. Dans le but de la valorisation de ces plantes, ce travail a pour objectif d'évaluer la relation composition chimique-activité antioxydante, et la toxicité des huiles essentielles de Cymbopogon citratus et Cymbopogon schoenantus acclimatées au Bénin. Les huiles essentielles (HE) ont été obtenues et analysées par $\mathrm{CPG} / \mathrm{DIF}$ et CPG/SM. Le test antioxydant a été réalisé par la méthode de DPPH et celui de la toxicité sur les larves de crevette de Artémia salina Leach. L'analyse chimique a révélé plusieurs composés dont le citral à $75 \%$ dans l'HE de Cymbopogon citratus et la pipéritone à 60,3\% dans celle de Cymbopogon schoenantus. L'activité antioxydante varie d'une plante à une autre avec l'huile de Cymbopogon schoenantus $\left(\mathrm{CE}_{50}=5040 \mu \mathrm{g} / \mathrm{mL}\right) \mathrm{plus}$ antioxydante que celle de Cymbopogon citratus $\left(\mathrm{CE}_{50}=47860 \mu \mathrm{g} / \mathrm{mL}\right)$. Cette activité de l'huile de Cymbopogon schoenantus pourrait s'expliquer par la présence de constituants comme la pipéritone et le limonène, reconnus pour leurs propriétés antioxydantes. Toutes les huiles étudiées sont moins antioxydantes que le composé de référence (acide ascorbique; $\mathrm{CE}_{50}=20 \mu \mathrm{g} / \mathrm{mL}$ ), et moins toxiques (CL50 $>30 \mu \mathrm{g} / \mathrm{mL}$ ) que la camptothécine (CL50 $=13,27 \mu \mathrm{g} / \mathrm{mL})$. Ces huiles essentielles, moins toxiques et déjà utilisées dans la médicine traditionnelle, pourraient également contribuer à la prise en charge des maladies dues aux radicaux libres.

(C) 2019 International Formulae Group. All rights reserved

Mots clés : Plantes aromatiques, composés volatils, Artémia salina Leach, antioxydants, médecine traditionnelle.

\section{Relationship between chemical composition, antioxidant activity and toxicity of essential oils from two species of Cymbopogon acclimated in Benin}

\begin{abstract}
Aromatic plants play a considerable role in several sectors, especially in traditional medicine. In order to promote these plants, this work aims at evaluating the relationship between chemical composition, antioxidant activity and the toxicity of the essential oils of Cymbopogon citratus and Cymbopogon schoenantus acclimated
\end{abstract}


in Benin. Essential oils (EO) were obtained and analyzed by GC/FID and GC/MS. The antioxidant test was carried out by the DPPH method and the toxicity test against shrimp larvae of Artemia salina Leach. Chemical analysis revealed several compounds including $75 \%$ of citral in Cymbopogon citratus EO and $60.3 \%$ of piperitone in Cymbopogon schoenantus one. The antioxidant activity varies according to the species with the oil of Cymbopogon schoenantus $\left(\mathrm{EC}_{50}=5040 \mu \mathrm{g} / \mathrm{mL}\right)$ more antioxidant than that of Cymbopogon citratus $\left(\mathrm{EC}_{50}=\right.$ $47860 \mu \mathrm{g} / \mathrm{mL}$ ). This activity of the Cymbopogon schoenantus oil could be explained by the presence of constituents such as piperitone and limonene, known to possess antioxidant properties. All the studied oils are less antioxidant than the reference compound (ascorbic acid, $\mathrm{EC}_{50}=20 \mu \mathrm{g} / \mathrm{mL}$ ), and less toxic $\left(\mathrm{LC}_{50}>30 \mu \mathrm{g} / \mathrm{mL}\right.$ ) than camptothecin $\left(\mathrm{LC}_{50}=13.27 \mu \mathrm{g} / \mathrm{mL}\right)$. These essential oils, less toxic and already used in traditional medicine, could also contribute to the management of diseases caused by free radicals.

(C) 2019 International Formulae Group. All rights reserved

Keywords: Aromatic plants, volatile compounds, Artemia salina Leach, antioxidants, traditional medicine.

\section{INTRODUCTION}

Depuis le $\mathrm{XVI}^{\mathrm{e}}$ siècle, les plantes aromatiques représentent une source de remèdes traditionnels et efficaces grâce aux principes actifs qu'elles renferment et qui se retrouvent dans leurs différents extraits comme les huiles essentielles. Le monde scientifique s'intéresse de plus en plus à ces dernières. Cymbopogon citratus (DC) Stapf et Cymbopogon schoenantus (L.) Spreng, deux espèces aromatiques du genre Cymbopogon, originaires d'Asie et appartenant à la famille des Poaceaes sont de sources potentielles d'huiles essentielles. Ces deux plantes sont répandues sur tous les continents et surtout utilisées du fait de leurs propriétés médicinales et culinaires (Cheel et al., 2005).

Traditionnellement, Cymbopogon citratus est utilisée contre des infections. Plantée, à proximité des maisons, cette plante est un antimoustique. De nombreuses propriétés sont reconnues pour son huile essentielle. Cette huile est antifongique, antiinflammatoire, antipyrétique, diurétique, analgésique, antispasmodique et sédative (Tiendrebeogo et al., 2017; Bassolé et al., 2011 ; Santin et al., 2009). Elle a également des propriétés antidiahérriques (Negrelle et Gomes, 2007; Tangpu et Yadav, 2006), antitrypanosomale et antiplasmodiale (Kpoviessi et al., 2014). Cette huile est constituée essentiellement du citral (néral et géranial) comme composé majoritaire mais aussi d'autres composés minoritaires. Ces constituants de l'huile essentielle présentent des concentrations qui varient d'un lieu à un autre (Diop et al., 2017; Kpoviessi et al., 2014 ; Nonviho et al., 2010).

Quant à Cymbopogon schoenantus, elle est utilisée traditionnellement, non seulement contre la fièvre et l'ictère (AL-Ali et al., 2017), mais aussi pour ses propriétés culinaire, antidiarrhéique, antirhumatismal, carminatif, diaphorétique, stomachique, diurétique etc (AL-Ali et al., 2017). Son huile essentielle possède des propriétés antimicrobiennes, antiprolifératives, antioxydantes, antifongiques (Yagi et al., 2016; Khadri et al., 2008); anticonvulsantes, insecticides, larvicides (Nonviho et al., 2010 ; Gbogbo et al., 2006). L'huile essentielle est constituée majoritairement de pipéritone, (+)-2-carène, limonène, élémol, etc (Yagi et al., 2016; Kpoviessi et al., 2014 ; Alitonou et al., 2012 ; Nonviho et al., 2010 ; Khadri et al., 2008). Selon la littérature, la composition chimique et les activités biologiques de cette huile varient selon le profil phénologique (KpadonouKpoviessi et al., 2012 ; Dabire et al., 2011).

Dans le but de valoriser ces deux plantes aromatiques acclimatées aux Bénin, le présent travail a pour objectif général d'étudier l'activité antioxydante et la toxicité des huiles essentielles de Cymbopogon citratus et de Cymbopogon schoenantus en relation avec leur composition chimique. 


\section{MATÉRIEL ET MÉTHODES \\ Matériel végétal}

Le matériel végétal était constitué de feuilles fraîches de Cymbopogon citratus (DC) stapf et de Cymbopogons schoenantus (L.) Spreng. Ces feuilles ont été récoltées en mars 2014 dans le jardin botanique de l'Université d'Abomey-Calavi (Bénin). Des spécimens ont été déposés après identification et authentification par l'Herbier National: $\mathrm{N}^{\circ}$ AA6387/HNB et $\mathrm{N}^{\circ}$ AA6390/HNB respectivement pour Cymbopogon citratus (DC) stapf et Cymbopogons schoenantus (L.) Spreng.

\section{Réactifs chimiques}

Les réactifs chimiques utilisés étaient le DPPH (2,2-Diphényl-1-picryl-hydrazyl), la (S)-(+)-camptothécine, l'acide ascorbique et le méthanol.

\section{Matériel biologique}

Les tests de toxicité étaient effectués sur des larves de crevette de saumure (Artemia salina Leach) obtenues après éclosion des œufs.

\section{Extraction des huiles essentielles}

L'extraction a été effectuée par hydrodiffusion avec un appareil de type Clevenger amélioré (Bruneton, 1993 ; Clevenger, 1928), pendant une durée de trois heures en général, pour chaque lot de récolte. L'huile essentielle, après décantation et séchage sur le sulfate de sodium, est récupérée dans un flacon puis conservée à $4{ }^{\circ} \mathrm{C}$ dans une chambre froide pour les analyses CPG/DIF et $\mathrm{CPG} / \mathrm{SM}$ et pour les tests biologiques.

\section{Analyse chimique des huiles essentielles}

L'analyse des huiles essentielles a été effectuée par la Chromatographie en phase Gazeuse avec détecteur à ionisation de flamme (CPG/DIF) et Chromatographie en phase Gazeuse couplée à la spectrométrie de masse
(CPG/SM) selon la méthode décrite précédemment par Kpoviessi et al. (2014).

\section{Test antioxydant}

La méthode par le DPPH a été utilisée pour l'évaluation de l'activité antioxydante des huiles. Dans une microplaque de 96 puits, une série de 10 dilutions successives (au 1/2) de chaque huile ont été réalisées à partir de solutions d'échantillon à $150 \mu \mathrm{L} / \mathrm{mL}$ dans le méthanol. Pour chaque concentration, trois (03) essais ont été effectués par ajout de $100 \mu \mathrm{L}$ de DPPH $(100 \mu \mathrm{g} / \mathrm{mL}$ dans le méthanol $)$ à toutes les dilutions, en cascade. Ainsi, le DPPH a été testé à une concentration unique de 50 $\mu \mathrm{g} / \mathrm{mL}$. La plaque a été incubée à l'obscurité pendant 20 minutes et l'absorbance lue à 517 $\mathrm{nm}$ à l'aide d'un spectrophotomètre. Le contrôle négatif était composé de $1 \mathrm{~mL}$ de la solution méthanolique et $1 \mathrm{~mL}$ de la solution de DPPH (100 mg/mL) (Otohinoyi et al., 2014 ; Brand-williams et al., 1995). Le contrôle positif était représenté par une solution d'un antioxydant standard; l'acide ascorbique (1 $\mathrm{mg} / \mathrm{mL}$ ) dont l'absorbance a été mesurée dans les mêmes conditions que l'échantillon testé.

L'activité antiradicalaire était estimée selon l'équation suivante :

$\%$ d'activité anti radicalaire $=$

absorbance (contrôle négatif)-absorbance (huile)

absorbance (contôle négatif)

La concentration d'extrait réduisant $50 \%$ de DPPH $\left(\mathrm{CE}_{50}\right)$ était obtenue avec le logiciel GraphPadPrism 4.0. Afin de mieux caractériser le pouvoir antiradicalaire avec le DPPH, le Pouvoir Antiradicalaire (PA) a été calculé par la formule :

$$
\mathrm{PA}=1 / \mathrm{CE}_{50}
$$

\section{Test de toxicité}

Le test que nous avons utilisé ici est le test de toxicité sur des larves de crevettes de saumures (Artemia salina Leach). C'est un test primaire de toxicité qui a été proposé par 
Awolola et al. (2010) et par Michael et al. (1956). Ce test consiste à mesurer la Concentration Létale à $50 \%\left(\mathrm{CL}_{50}\right)$ qui est la concentration à partir de laquelle au moins cinquante pour cent des larves de crevettes contenues dans le milieu de culture (milieu réactionnel) sont tuées.

\section{Mode opératoire}

Les œufs de Artenia salina étaient incubés dans de l'eau de mer durant 48 heures, temps nécessaire pour qu'ils éclosent afin de donner naissance à de jeunes larves. On prépare ensuite une série de milieux de concentrations progressives de l'échantillon d'huile essentielle à tester, dans lesquels on plonge un nombre identique, seize (16), de larves vivantes. Les milieux et les témoins ne contenant pas d'huile essentielle étaient laissés sous agitation pendant 24 heures. Après ces 24 heures, le comptage visuel microscopique a permis la détermination du nombre de larves mortes dans chaque milieu. Dans le cas où des décès étaient constatés dans le milieu témoin, les données étaient corrigées par la formule d'Abbott :

$$
\% \text { décès }=[(\text { test }- \text { témoin }) / \text { témoin })] \mathrm{x}
$$
100 (Abbott, 1925).

Les données dose-réponse ont été transformées en pourcentage (\%) d'inhibition et la concentration létale était déterminée avec le logiciel GraphPadPrism 4.0.

\section{Analyse statistique}

Le $t$-test de Student a été utilisé afin de déterminer si la différence entre les résultats obtenus pour les différents échantillons, et celle entre les résultats des échantillons et les contrôles sont significatives (GraphPadPrism 4.0 ; GraphPad Software Inc., San Diego, USA). La différence statistiquement significative était fixée à p < 0,05 (SAS/STAT, 1990 ; Steel et Torrie, 1980).

\section{RESULTATS}

\section{Rendement et composition chimique}

Les huiles essentielles sont obtenues avec un rendement de $0,71 \%$ et $1,88 \%$ respectivement pour Cymbopogon citratus et Cymbopogon schoenantus (Tableau 1). Les analyses par chromatographie en phase gazeuse couplée à la spectrométrie de masse (CPG/SM) et par chromatographie en phase gazeuse avec détection à ionisation de flamme (CPG/DIF) ont permis d'élucider la composition chimique des huiles essentielles de ces deux plantes étudiées (Tableau 1).

\section{Activité antioxydante des huiles essentielles}

L'activité antioxydante et le pouvoir antiradicalaire, exprimés respectivement en termes de $\mathrm{CE}_{50}$ et de PA, sont consignés dans le Tableau 2. L'analyse de ce Tableau montre que les échantillons d'HE présentent d'activité antioxydante. L'activité antioxydante de l'huile de Cymbopogon citratus est de 47860 $\mu \mathrm{g} / \mathrm{mL}$ et celle de l'huile de Cymbopogon schoenantus est de $5040 \mu \mathrm{g} / \mathrm{mL}$.

\section{Toxicité des huiles essentielles}

Les huiles essentielles étudiées sont testées pour leur toxicité sur les larves de crevettes (Artemia salina L.). Le test utilisé ici est une méthode préliminaire de toxicité in vitro qui n'est pas totalement superposable à un test sur des cellules humaines en culture. Bien qu'il y ait une corrélation entre les deux types de cellules, Carballo et al. (2002) suggérèrent d'effectuer les deux tests avant de conclure ; ce qui donnerait une fiabilité de $75 \%$ contre $50 \%$ pour le test sur les larves de crevettes seules. Les résultats exprimés en $\mathrm{CL}_{50}$ sont consignés dans le Tableau 2. La toxicité $\left(\mathrm{CL}_{50}\right)$ de l'huile de Cymbopogon citratus est de $64,29 \mu \mathrm{g} / \mathrm{mL}$ et de $30,19 \mu \mathrm{g} / \mathrm{mL}$ pour celle de Cymbopogon schoenantus. 
Tableau 1 : Composés majoritaires des HE de C. citratus et C. schoenantus.

\begin{tabular}{|c|c|c|c|}
\hline Composés & IK & C. citratus & C. schoenantus \\
\hline$\beta$-Pinène & 996 & $10,1 \pm 0,04$ & \\
\hline (b)-2-Carène & 1005 & - & $13,0 \pm 0,20$ \\
\hline Limonène & 1028 & - & $6,4 \pm 0,10$ \\
\hline cis-verbénol & 1199 & $1,7 \pm 0,01$ & - \\
\hline Néral & 1268 & $35,5 \pm 0,15$ & - \\
\hline cis-géraniol & 1291 & $4,3 \pm 0,02$ & - \\
\hline Pipéritone & 1296 & & $60,3 \pm 0,92$ \\
\hline Géranial & 1328 & $39,5 \pm 0,00$ & - \\
\hline Elémol & 1556 & - & $4,9 \pm 0,08$ \\
\hline$\beta$-Eudesmol & 1648 & - & $3,1 \pm 0,05$ \\
\hline Rendement (\%) & & $0,71 \pm 0,02$ & $1,88 \pm 0,12$ \\
\hline
\end{tabular}

C. : Cymbopogon ; IK : Indice de Kovate ; HE : Huile Essentielle. Les valeurs sont des moyennes \pm écart type de trois expériences différentes.

Tableau 2 : Activité antioxydante et toxicité des HE de C. citratus et C. schoenantus.

\begin{tabular}{llll}
\hline & $\begin{array}{l}\text { Activité } \\
\text { antioxydante en CE50 } \\
(\boldsymbol{\mu g} / \mathbf{m L})\end{array}$ & $\begin{array}{l}\text { Pouvoir Anti } \\
\text { Radicalaire en } \\
\text { PA }\left(\mathbf{1 0}^{-5}\right)\end{array}$ & $\begin{array}{l}\text { Toxicité en CL50 } \\
(\boldsymbol{\mu g} / \mathbf{m L})\end{array}$ \\
\hline C. citratus & $47860 \pm 0,00^{\mathrm{c}}$ & 2,08 & $64,29 \pm 0,01^{\mathrm{c}}$ \\
C. schoenantus & $5040 \pm 0,00^{\mathrm{b}}$ & 19,84 & $30,19 \pm 0,03^{\mathrm{b}}$ \\
Acide ascorbique & $20 \pm 0,00^{\mathrm{a}}$ & 5000 & $\mathrm{nd}$ \\
Camptothécine & nd & nd & $13,27 \pm 0,02^{\mathrm{a}}$ \\
\hline $\begin{array}{l}\text { nd : non déterminé, } C .: \text { Cymbopogon, Les données de la même colonne suivies de lettres différentes }(\mathrm{a}, \mathrm{b}, \mathrm{c}, \ldots) \text { sont } \\
\text { statistiquement différentes selon le } t \text {-test de Student }(\mathrm{p}=0,05) . \text { Les valeurs sont des moyennes } \pm \text { écart type de trois } \\
\text { expériences différentes. }\end{array}$
\end{tabular}

\section{DISCUSSION}

\section{Rendement et composition chimique}

Les résultats de rendement en huile essentielle obtenus montrent que le rendement varie d'une plante à une autre. Ces résultats sont conformes à celui décrit précédemment par Kpoviessi et al. (2014) dans la même localité. Ils sont différents de ceux obtenus par Nonviho et al. (2010) qui sont $1,7 \%$ pour
Cymbopogon citratus à Akogbato (sud Bénin) et 2,8\% pour Cymbopogon schoenantus à Boukoumbé (Nord Bénin) et de celui obtenu par Alitonou et al. (2012) pour l'huile essentielle de Cymbopogon schoenantus $(3,49 \%)$ récolté à Djougou (Nord Bénin). Ces auteurs ont travaillé sur le matériel séché. La différence observée au niveau des rendements pourrait s'expliquer, d'une part, par l'influence 
de l'état (frais ou sec) du matériel utilisé (Kpadonou-Kpoviessi et al., 2012 ; Dabire et al., 2011 ; Barbosa et al., 2008) et, d'autre part, par la différence du lieu de récolte (Onadja et al., 2007). Les résultats de l'analyse chimique par $\mathrm{CPG} / \mathrm{DIF}$ et $\mathrm{CPG} / \mathrm{SM}$ sont conformes à ceux publiés précédemment par Kpoviessi et al. (2014) et Nonviho et al. (2010). Les légères différences pourraient s'expliquer par des facteurs comme: le temps et le lieu de récolte, le temps d'extraction et le stade végétatif qui peuvent influencer la composition chimique de l'HE d'une plante (Kpadonou-kpoviessi et al., 2012; Barbosa et al., 2008). Les cinq composés majoritaires de chacune des huiles étudiées dans le présent travail sont consignés dans le Tableau 1.

\section{Activité antioxydante des huiles essentielles}

Les résultats de l'activité antioxydante et du pouvoir antiradicalaire montrent que les échantillons d'huile essentielle présentent l'activité antioxydante qui varie d'une plante à une autre, malgré l'appartenance au même genre Cymbopogon de ces deux plantes. L'activité de l'huile de Cymbopogon schoenantus $\left(\mathrm{CE}_{50}=5040 \mu \mathrm{g} / \mathrm{mL}\right)$ est au moins neuf (9) fois plus intéressante que celle de Cymbopogon citratus $\left(\mathrm{CE}_{50}=47860 \mu \mathrm{g} / \mathrm{mL}\right)$. L'activité antioxydante de ces huiles pourrait s'expliquer par la présence de certains composés reconnus pour leur activité antioxydante tels que la carvone $\left(\mathrm{CE}_{50}=32100\right.$ $\mu \mathrm{g} / \mathrm{mL}), l^{\prime} \alpha$-terpinéol $\left(\mathrm{CE}_{50}=332800 \mu \mathrm{g} / \mathrm{mL}\right)$ (Bicas et al., 2011). La forte activité antioxydante de l'huile de Cymbopogon schoenantus pourrait s'expliquer par la structure de la pipéritone, composé de plus de $60 \%$ d'huile, qui comporte une double liaison $\mathrm{C}=\mathrm{C}$ conjuguée à un groupe carbonylé, de même qu'il en ait dans la structure de carvone (Bicas et al., 2011). Aussi le limonène (6,4\%), un monoterpène hydrocarboné de l'huile, contribuerait à l'amélioration de l'activité antioxydante car il est un antistress significatif, à actions mesurables par des paramètres comportementaux et physiologiques, sous l'influence du système nerveux (Alessio et al., 2014). L'activité de l'huile essentielle de Cymbopogon citratus, s'expliquerait aussi par la présence d'aldéhydes et d'alcools (citral constituant majoritaire, géraniol...), qui utilisent divers mécanismes dans le processus de désactivation des radicaux (Bayala et al., 2018). L'activité antioxydante de nos huiles est plus faible que celle de l'acide ascorbique, composé de référence $\left(\mathrm{CI}_{50}=20 \mu \mathrm{g} / \mathrm{mL}\right)$. Ces plantes médicinales constitueraient alors une source de traitement des maladies.

\section{Toxicité des huiles essentielles}

Les résultats de toxicité prouvent que la toxicité larvaire des huiles varie d'une plante à une autre. Pour l'huile de Cymbopogon citratus, la valeur est de $64,29 \mu \mathrm{g} / \mathrm{mL}$ et de $30,19 \mu \mathrm{g} / \mathrm{mL}$ pour celle de Cymbopogon schoenantus. L'huile de Cymbopogon citratus, est au moins deux fois moins toxique que celle de Cymbopogon schoenantus. Toutes les valeurs obtenues sont supérieures à $30 \mu \mathrm{g} / \mathrm{mL}$ et sont significativement différentes selon le ttest de Students $(p=0,001)$ de celle de la camptothécine, composé de référence $\left(\mathrm{CL}_{50}\right.$ $=13,27 \mu \mathrm{g} / \mathrm{mL}$ ). Nos échantillons d'huiles essentielles sont alors moins toxiques. Afin de confirmer la toxicité ou non de ces huiles, il serait important d'effectuer des tests in vivo. Ce qui expliquerait l'utilisation de ces plantes sans grands risques d'intoxication dans la médecine traditionnelle. Ce travail éclaire quant à l'innocuité de ces huiles essentielles. Seulement, il conviendra de respecter les limites de dosage dans son administration.

\section{Conclusion}

Les huiles essentielles de Cymbopogon citratus et Cymbopogon schoenantus, deux plantes acclimatées au Bénin, présentent une activité antioxydante qui varie d'une plante à une autre, et en fonction de la composition chimique. De même, leur toxicité varie. L'activité antioxydante de ces HE est plus faible que celle de l'acide ascorbique, le contrôle positif. Les huiles sont moins toxiques que la camptothécine, composé de référence. L'activité antioxydante et la faible toxicité de ces huiles, confirment leur usage dans la médecine traditionnelle. Il serait donc intéressant d'effectuer des études in vivo afin 
d'apporter des réponses plus appropriées aux questions concernant l'innocuité de ces huiles.

\section{CONFLIT D'INTERETS}

Les auteurs déclarent qu'il n'y a pas de conflit d'intérêts.

\section{CONTRIBUTIONS DES AUTEURS}

Chaque auteur a contribué équitablement.

\section{REMERCIEMENTS}

Les auteurs remercient le Professeur Joëlle Quetin-Leclercq pour avoir permis l'analyse chromatographique des huiles essentielles dans son laboratoire. Ce travail a été soutenu en partie par le projet VALTRAMED (ARES-CCD).

\section{REFERENCES}

Abbott WS. 1925. A method of computing the effectiveness of an insecticide. J. Econ. Entomol., 18(2): 265-267. DOI: https://doi.org/10.1093/jee/18.2.265a.

AL-Ali KH, Ibrahim NA, Abdulrahman Ahmed AA, Hemeg HA, Abdelgawwad MA, Abdel-Salam HA. 2017. Evaluation of the antimicrobial activities of Cymbopogon schoenanthus. Afr. J. Microbiol. Res., 11(17): 653-659. DOI: https://doi.org/10.5897/AJMR2017.8554

Alessio PA, Bisson J-F, Béné MC. 2014. Antistress effects of d-limonene and its metabolite perillyl alcohol, Rejuvenation Res., 17(2): 145-149. DOI: https:// doi.org/10.1089/rej.2013.1515.

Alitonou GA, Avlessi F, Tchobo F, Noudogbessi J-P, Tonouhewa A, Yehouenou B, MENUT C, Sohounhloue DK. 2012. Chemical composition and biological activities of essential oils from the leaves of Cymbopogon giganteus Chiov. and Cymbopogon schoenanthus (L.) Spreng (Poaceae) from Benin. Int. J. Biol. Chem. Sci., 6(4): 1819-1827. DOI: http://dx.doi.org/10.4314/ijbcs.v6i4.37.
Awolola GV, Oluwaniyi OO, Solanke A, Dosumu OO, Shuiab AO. 2010. Toxicity assessment of natural and chemical coagulants using brine shrimp (Artemia salina) bioassay. Int. J. Biol. Chem. Sci., 4(3): 633-641.

Barbosa LCA, Pereira UA, Martinazzo AP, Maltha CRA, Teixeira RR, de Castro Melo E. 2008. Evaluation of the Chemical Composition of Brazilian Commercial Cymbopogon citratus (D.C.) Stapf Samples. Molecules., 13(8): 1864-1874. DOI: https://doi.org/10.3390/molecules 130818 64

Bassolé IHN, Lamien-Meda A, Bayala B, Obame LC, Ilboudo AJ, Franz C, Novak J, Nebié RC, Dicko MH. 2011. Chemical composition and antimicrobial activity of Cymbopogon citratus and Cymbopogon giganteus essential oils alone and in combination. Phytomedicine, 18(12): 1070-1074. DOI: https://doi.org/10.1016/j.phymed.2011.0 5.009

Bayala B, Bassole IHN, Maqdasy S, Baron S, Simpore J, Lobaccaro JA. 2018. Cymbopogon citratus and Cymbopogon giganteus essential oils have cytotoxic effects on tumor cell cultures. Identification of citral as a new putative anti-proliferative molecule. Biochimie, 153: $162-170 . \quad$ DOI : https://doi.org/10.1016/j.biochi.2018.02. 013

Bicas JL, Neri-Numa IA, Ruiz ALTG, De Carvalho JE, Pastore GM. 2011. Evaluation of the antioxidant and antiproliferative potential of bioflavors. Food Chem. Toxicol., 49(7): 1610-1615. DOI : https://doi.org/10.1016/j.fct.2011.04.012. Brand-williams W, Cuvelier ME, Brest C. 1995. Use of a method to evaluate antioxidant actvity. Lebensm. Wiss. Technol., 28: 25-30. DOI : https://doi.org/1016/SOO236438(95)80008-5. 
Bruneton J. 1993. Pharmacognosie: Phytochimie, Plantes Médicinales (2 $2^{\mathrm{ème}}$ édition). Technique et DocumentationLavoisier : Paris ; 387-404.

Carballo JL, Zaira L, Indal H, Pilar P, GarciaGravalos MD. 2002. Comparaison between two brine shrimp assays to detect in vitro cytotoxicity in marine natural products. BioMed Central Biotechnology, 2 : 17. DOI : https://doi.org/10.1186/1472-6750-2-17.

Cheel J, Theoduloz C, Rodriquez J, ShemedaHirshmann G. 2005. Free radical scavengers and antioxidant from lemon grass (Cymbopogan citratus). $J$. Agric. Food Chem., 53: 2511-2517. DOI: https://pubs.acs.org/doi/abs/10.1021/jf04 79766

Clevenger JF. 1928. Apparatus for the deterioration of volatile oil. $J$. Am. Pharm. Assoc., 17(4) : 345-349. DOI : https://doi.org/10.1002/jps.30801717040 7

Dabire C, Nebié RHC, Belanger A, Nacro M, Sib FS. 2011. Effet du séchage de la matière végétale sur la composition chimique de l'huile essentielle et l'activité antioxydante d'extraits d'Ocimum basilicum L. Int. J.Biol. Chem. Sci., 5(3) : 1082-1095.

DOI : http://dx.doi.org/10.4314/ijbcs.v5i3.7221 8

Diop SM, Guèye MT, Ndiaye I, Diop MB, Ndiaye B, Thiam A, Fauconnier M-L, Lognay G. 2017. Study of the chemical composition of essential oils and floral waters of Cymbopogon citratus (DC.) Stapf (Poaceae) from Senegal. Int. J. Biol. Chem. Sci., 11(4): 1884-1892. DOI: http://dx.doi.org/10.4314/ijbcs.v11i4.37

Gbogbo KA, Batawila K, Anani K, PrinceDavid M, Gbéassor M, Bouchet $\mathrm{P}$, Akpagana K. 2006. Activité antifongique des huiles essentielles de Ocimum basilicum L. (Lamiaceae) et Cymbopogon schoenanthus (L.) Spreng. (Poaceae) sur des micromycètes influençant la germination du Maïs et du Niébé. Acta botanica Gallica., 153(1): 115-124. DOI : http://dx.doi.org/10.1080/12538078.2006 .1051552

Khadri A, Serralheiro MLM, Nogueira JMF, Neffati M, Smiti S, Araújo MEM. 2008. Antioxidant and antiacetylcholinesterase activities of essential oils from Cymbopogon schoenanthus L. Spreng. Determination of chemical composition by GC-mass spectrometry and 13C NMR. Food Chem., 109(3): 630-637. DOI https://doi.org/10.1016/j.foodchem.2007. 12.070

Kpadonou-Kpoviessi BGH, Yayi-Ladekan E, Kpoviessi DSS, Gbaguidi F, Yehouenou B, Quetin-Leclercq J, Figueredo G, Moudachirou M, Accrombessi GC. 2012. Chemical variation of essential oil constituents of Ocimum gratissimum L. from Benin, and impact on antimicrobial properties and toxicity against Artemia salina Leach. Chem. Biodivers., 9(1): 139-150.

DOI : https://doi.org/10.1002/cbdv.201100194

Kpoviessi S, Bero J, Agbani P, Gbaguidi F, Kpadonou-Kpoviessi B, Sinsin B, Accrombessi S, Frédérich M, Moudachirou M, Quetin-Leclercq J. 2014. Chemical composition, cytotoxicity and in vitro antitrypanosomal and antiplasmodial activity of the essential oils of four Cymbopogon species from Benin. $J$ Ethnopharmacol., 151(1): 652-659. DOI: https://doi.org/10.1016/j.jep.2013.11.027

Michael AS, Thompson CG, Abramovitz M. 1956. Artemia salina as a test organism for a bioassay. Science, 123(3194): 464. DOI: https://doi.org/10.1126 / science.123.3194.464.

Negrelle RRB, Gomes EC. 2007. Cymbopogon citratus (DC.) Stapf: chemical composition and biological activities. Rev. Bras. Pl. Med., 9: 80-92.

Nonviho G, Wotto VD, Noudogbessi JP, Avlessi F, Akogbeto M, Sohounhloué DCK. 2010. Insecticidal activities of essential oils extracted from three species of Poaceae on Anopheles gambiae SPP, major vector of malaria. Sci Study Res 
Chem Chem Eng Biotechnol Food Ind., 11(4): 411- 420.

Onadja Y, Ouedraogo A, Samate AD. 2007. Chemical composition and physical characteristics of the essential oil of Cymbopogon schoenanthus (L.) Spreng of Burkina Faso. J. Appl. Sci., 7(4): 503506. DOI: https:// doi.org/10.3923/jas.2007.503.506

Otohinoyi DA, Ekpo O, Ibraheem O. 2014. Effect of ambient temperature storage on 2,2-diphenyl-1-picrylhydrazyl (DPPH) as a free radical for the evaluation of antioxidant activity. Int. J. Biol. Chem. Sci., 8(3): 1262-1268. DOI: http://dx.doi.org/10.4314/ijbcs.v8i3.39

Santin MR, Dos Santos AO, Nakamura CV, Dias Filho BP, Ferreira ICP, UedaNakamura T. 2009. In vitro activity of the essential oil of Cymbopogon citratus and its major component (citral) on Leishmania amazonensis. Parasitol. Res., $\quad$ 105(6): 1489-1496. DOI : https://doi.org/10.1007/s00436009-1578-7
SAS/STAT. 1990. User'Guide. SASInstitute, Inc. SAS Institute ( $4^{\text {th }}$ edn). SAS Inst., Inc., Cary; NC., 2(6); 951-958.

Steel RGD, Torrie JH. 1980. Principales and Procedures of Statistics ( $2^{\text {nd }}$ edn). McGraw-Hill: New York.

Tangpu V, Yadav AK. 2006. Antidiarrhoeal activity of Cymbopogon citratus and its main constituent, citral. Pharmacologyonline, 2: 290-298.

Tiendrebeogo A, Ouedraogo I, Bonzi S, Kassankogno AI. 2017. Etude de l'activité antifongique d'extraits de Cymbopogon citratus (DC.) Stap, Eclipta alba L., Lippia multiflora M. et Agave sisalana P. Int. J. Biol. Chem. Sci., 11(3): 1202-1211.

DOI: https://dx.doi.org/10.4314/ijbcs.v11i3.22

Yagi S, Babiker R, Tzanova T, Schohn H. 2016. Chemical composition, antiproliferative, antioxidant and antibacterial activities of essential oils from aromatic plants growing in Sudan. Asian Pac. J. Trop. Med., 9(8): 763-770. DOI: https://doi.org/10.1016/j.apjtm.2016.06.0 09. 